\title{
Distributed Signal Processing Algorithms for Wireless Sensor Networks
}

\author{
Ashwini S Chiwarkar \\ Department of Electronics \& Telecommunication \\ Engineering \\ Shri Sant Gajanan Maharaj College of Engineering, \\ Shegaon, Maharashtra, India
}

\begin{abstract}
Wireless Body Area Networks (WBAN), in particular in the field of wearable health monitoring system (WMB), such as electromagnetic cardiograms (ECG) data collecting system via WBANs in e-health applications, is becoming increasingly important for future communication systems. Compressive sensing (CS), on the other hand, has been shown to consume less power compared classic transform-coding-based approaches. We propose a new low-rank sparse deep signal recovery algorithm for recovering ECG data in the context of CS (Compressive sensing) because the spatial and temporal data collected by a WBAN have some closely correlated structures in certain wavelet domains e.g., the discrete wavelet transform (DWT) domain.
\end{abstract}

Keywords: Wireless Sensor Network, WBAN, Distributed Signal Processing, Compressive sensing.

\section{INTRODUCTION}

For statistical inference in wireless connections, distributed signal processing techniques are used. Utilizing distributed analysis methods, data is extracted from data gathered at nodes dispersed along a geographic region. A group of surrounding nodes collects nearby data for each node, together including their local estimations for a better estimate, and sends it to each node [1][2].

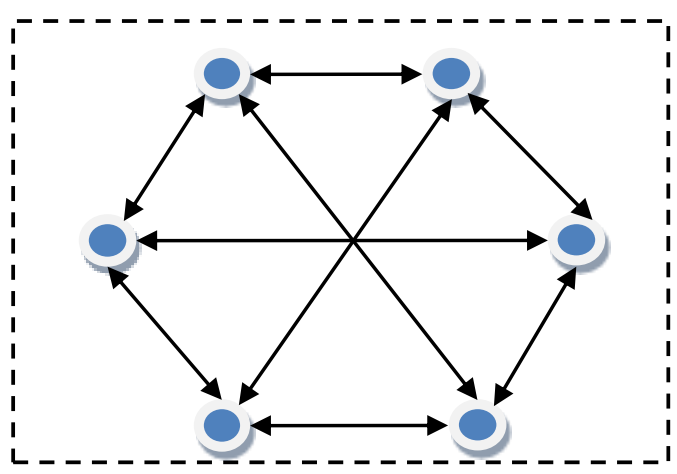

Figure 1. Distributed wireless network

Distributed signal processing (DSP) is a networking design and evaluation approach that may solve optimization and adaptation issues in a distributed and productive way [3].

\author{
Dr. K. B. Khanchandani \\ Department of Electronics \& Telecommunication \\ Engineering \\ Shri Sant Gajanan Maharaj College of Engineering, \\ Shegaon, Maharashtra, India
}

\subsection{Distributed Wireless Networks}

A WLAN network allows a WLAN hotspot to be extended along a greater geographical region without any need for cables to be attached to each access point (AP). It is comprised of two or even more Wi-Fi (access point) base stations that work together as one platform. The backbone will be utilised as a backbone for computers, cell telephones, monitors, controllers, and processors in upcoming data transmission in distributed wireless networks. In this scenario, the dispersed networks has no central controller, causing the entire network to collapse. Furthermore, in a dispersed network, many data transmission paths are possible.

Distributed Estimation - In the distributed parameters estimation, we concentrate on processing an unknown vector 0 of size $\mathrm{M} \times 1$. The intended signal for each node at the point in a given time $\mathrm{i}$ is

$$
d_{k}(i)=\omega_{0}{ }^{H} x_{k}(i)+n_{k}(i), i=1,2, \ldots, I
$$

The result of the adaptive algorithm for every node is provided by

$$
\left.y_{k}(i)=\omega_{k}{ }^{H} x_{k}(i)\right), i=1,2, \ldots, I
$$

A problem described in the form of a minimizing cost in the distributing format at each node $\mathrm{k}$ is used to predict unknown vector

$$
\mathrm{J}_{\mathrm{k}}\left(\omega_{\mathrm{k}}(\mathrm{i})\right)=\mathrm{E}\left|\mathrm{d}_{\mathrm{k}}(\mathrm{i})-\omega_{\mathrm{k}}{ }^{\mathrm{H}} \mathrm{x}_{\mathrm{k}}(\mathrm{i})\right|^{2}
$$

the global network cost function could be described as

$$
\mathrm{J}_{\mathrm{k}}\left(\omega_{\mathrm{k}}(\mathrm{i})\right)=\sum_{k=1}^{N} \mathrm{E}\left|\mathrm{d}_{\mathrm{k}}(\mathrm{i})-\omega_{k}^{H} \mathrm{x}_{\mathrm{k}}(\mathrm{i})\right|^{2}
$$

\subsection{Compressive Sensing Techniques}

5G wireless communication technologies are intended to power the next generation Internet of Things (IoT). In view of the growing number of Internet-of-things [1] devices, wireless IoT will become dense in the near future, taking place a tremendous variety of complicated exchanges and turning into self-organized systems [4]-[10]. For instance, contemporary advances in industrial automation approaching Industry 4.0 
models drive factory transformations into extremely flexible and recognizable manufacturing processes. For instance, contemporary advances in industrial automation approaching Industry 4.0 models drive factory transformations into extremely flexible and recognizable manufacturing processes. Radio technologies also can only play an important part in this context when combined with modern systems to allow ultrareliable Low Latency (URLL), decentralized computing capabilities and massive machine communication (mMTC), for self-organizing essential applications [11].

Current technology is confronted with an expanding amount of information in the era of the Internet of Things, and the increasing amount of information of all types, as well as the growing capabilities of modern gadgets in the processing of high-quality information. These massive amounts of data must be

- $\quad$ sensed like signal acquisition

- transmitted like telecommunication technology

- processed.

All three functions, notably sensing, storage memory, high transition bandwidth, necessitate data processing, and of course, greater power. Compression was proposed and applied in the field of signals and images, in which a signal/image was altered and saved in a considerably smaller size. When the compressed signal/image was needed, it was decompressed to its original size. Compressive sensing was proposed to prevent unnecessarily sampling redundant data and bearing the aforementioned adverse effect [12].

The signal is sampled through compressive sensing with a substantially fewer amount of trials than the Nyquist-Shannon theorem requires. It is predicated on the premise that the signal is sparse. Naturally, this approach assumed true for the vast majority of data types found in nature. Compressive sensing mainly is a challenge to

- compressively measure a signal while its information content is kept preserved

- $\quad$ to recover the original signal after compressive sensing. Compressive methods have a wide range of applications, including signal processing, smart environments, telecommunications, acoustic OFDM.

In its most basic form, compressive sensing is modeled as follows:

Consider $\mathrm{x}$ to be a signal vector with length $\mathrm{n}$ in the $\mathrm{Rn}$ vector space. Then $\mathrm{x}$ can be compressed felt, or displayed as a vector $\mathrm{y}$ of length $\mathrm{m}$ in the vector space $\mathrm{Rm}$, as follows:

$$
\mathrm{Y}_{\mathrm{m} \times 1}=\mathrm{A}_{\mathrm{m} \times \mathrm{n}} * \mathrm{X}_{\mathrm{n} \times 1}
$$

The name "compressive sensing" comes from the fact that $\mathrm{m}$ is substantially shorter than $\mathrm{n}, \mathrm{m} \mathrm{n}$. A compressive sensing matrix is what it's called. A long signal vector is sensed as a much shorter signal vector in compressive sensing, as shown in Fig 2.

\section{LITERATURE REVIEW}

Some research has been done into the usability and structure of CS when utilized for signal acquisition in WBAN. Mamaghanian et al. [2] evaluated the efficiency of CS for ECG capture in detail, as previously stated. Lastly, the energy savings were enhanced to 52.04 percent. The CS acquisition of non invasive baby ECG, which is a key branch in healthcare systems and can be employed for the identification of embryonic development and behavior, was also studied by the researchers. The downsides of fetal ECG, such as strong noise and non-sparsity, which are incompatible with standard CS frameworks, have been well remedied by sparse Bayesian learning; raw foetal ECG recordings are recreated with acceptable quality while synchronously maintaining interconnectedness relationships of multi-channel signals.

Brunelli and Caione [3] investigated the energy usage issue of both digital and analog CS, conducting a valid assessment utilizing real resource-constrained hardware architecture to investigate the influence of CS variable on signal recovery performance and sensor longevity.

Majumdar and Ward [4] integrate state-of-the-art blind CS and low-rank approaches, and then create a Split Bregman strategy to address the EEG signal restoration issue in WBAN.

In a rakeness-based CS structure with a zeroing approach, Mangia et al. [5] examined tradeoffs among data compression and signal reconstructing. The potential of CS for signal acquisition in IoT has been widely investigated in terms of sensor, transmitting, and reconstructing to relax energy usage and extend network capacity by establishing revolutionary cluster-sparse signal reconstruction methods.

Furthermore, to the consumption-efficient signal sampling technique, Peng et al. [6] considered secure transmission of the received signals in WBAN. In order to construct a joint signal capture, compressing, and encrypting system, chaos theory was added into the CS network to obtain a secret measurement matrix. Such a concept demonstrates chaos-based CS's huge potential for secure CS in WBAN and other IoT applications. The sliding window treatment method was also abandoned in order to make significant advances in signal recovery while lowering communication needs.

\section{METHODOLOGY}

By employing, the concept of sparsely, CS develops as a novel framework for signal collection. Rather than using the usual sampling and compression data-gathering method, CS shows how the number of data points necessary can be drastically decreased if the signal is sparse or compressing in some way. In this case, CS collects the information directly, compressive and at a lesser pace. Figure 4.9 depicts the sampling method, which is discussed below. Suppose that the signal to be sampled is k-sparse and column vector $x=$ $\left(x_{1}, x_{2}, \ldots \ldots . x_{N}\right)^{t}$, under the basis $\psi$ and that it is represented as

$$
x=\psi S
$$

Here, $\left\|S_{0}\right\|=\#\left\{i: S_{i} \neq 0\right\}=k \ll N$.

$y=\left(y_{1}, y_{2}, \ldots . y_{N}\right)^{T}$ is the evaluation vector; in other terms, the CS sampling product is the internal multiplication of $\mathrm{x}$ with a specific function. 


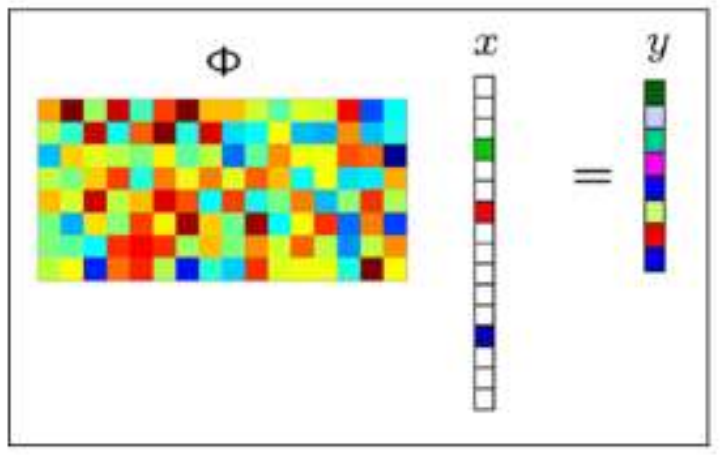

Figure 2: Sampling Process of CS

$$
y=\varphi x=\varphi \psi S
$$

where $\mathrm{M}=\mathrm{O}(\mathrm{k} \cdot \log (\mathrm{N} / \mathrm{k})) \mathrm{N}$ pre-determined projecting vectors make up the measurement matrix (1). In comparison to the Nyquist sampling theorem, it is evident that $\mathrm{M} \mathrm{N}$ denotes a decrease in dimension, and so CS denotes a reduced sampling frequency. Where, $m=o\left(k \cdot \log \left(\frac{N}{k}\right) N\right)$ predetermined projecting vectors make up the measurement matrix $(\varphi)$. In comparison to the Nyquist sampling theorem, it is evident that $m<N$ denotes a decrease in dimension, and so CS denotes a reduced sampling frequency. To summarize, in the CS procurement process, the old method of high-frequency sampling using Nyquist theory and then data compression using the sparse transformation has been substituted by a simple low-rate linear projection. As a result, system complexity reduction can be achieved and energy efficiency can be enhanced.In the Nyquist sampling theorem, signal restoration is done via linear sinc interpolation, that does not match the CS architecture, which is defined by an easily interpretable linear system. The inherent nonlinear technique solves the following optimization issue by exploiting the sparsity constraint.

$$
\min \|s\|_{0} \text { subject to } y=\varphi \psi S=\odot S
$$

The actual signal $\mathrm{x}$ is then retrieved using $x=\psi S$. Addressing equation demands thoroughly searching all $\mathrm{k}$ sub-columns from $\mathrm{N}$ ones, and it is determined to be a non-deterministic polynomial-time (NP)-hard task for normal-sized signals. The convex relaxed expression of equation is referred to as the alternate reconstructive approach.

$$
\min \|s\|_{1} \text { subject to } y=\odot S
$$

To ensure that this optimization issue has a distinctive and consistent solutions, the sensing matrix $\odot$ must satisfy the restrictive isometric property (RIP) of order $2 \mathrm{k}$, which means that there must be a constant $\mu_{2 k} \in[0,1]$ such that

$$
\left(1-\mu_{2 k}\right)\|x\|^{2} \leq\|\odot x\|^{2} \leq\left(1+\mu_{2 k}\right)\|x\|^{2}
$$

The following theorem dominates the precision of restoration for any $2 \mathrm{k}$-sparse signals $\mathrm{x}$.

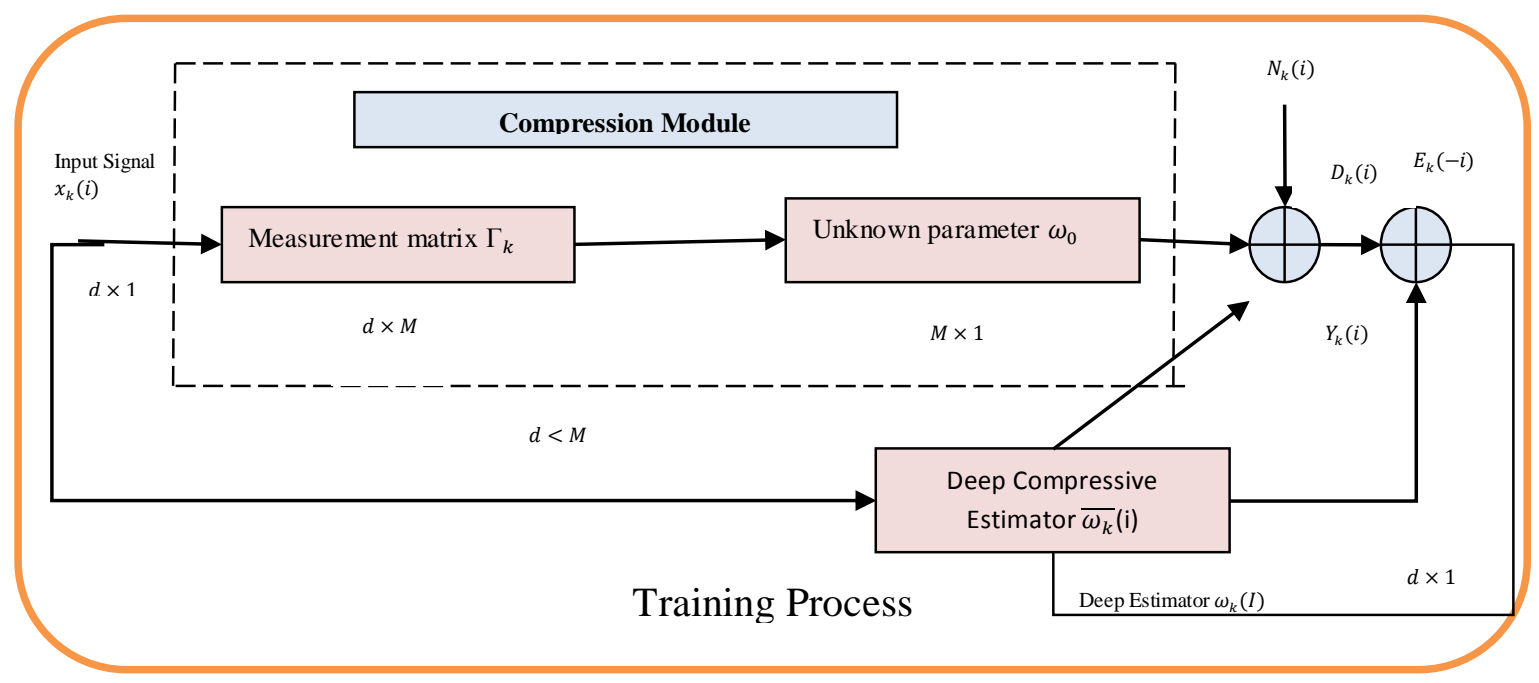




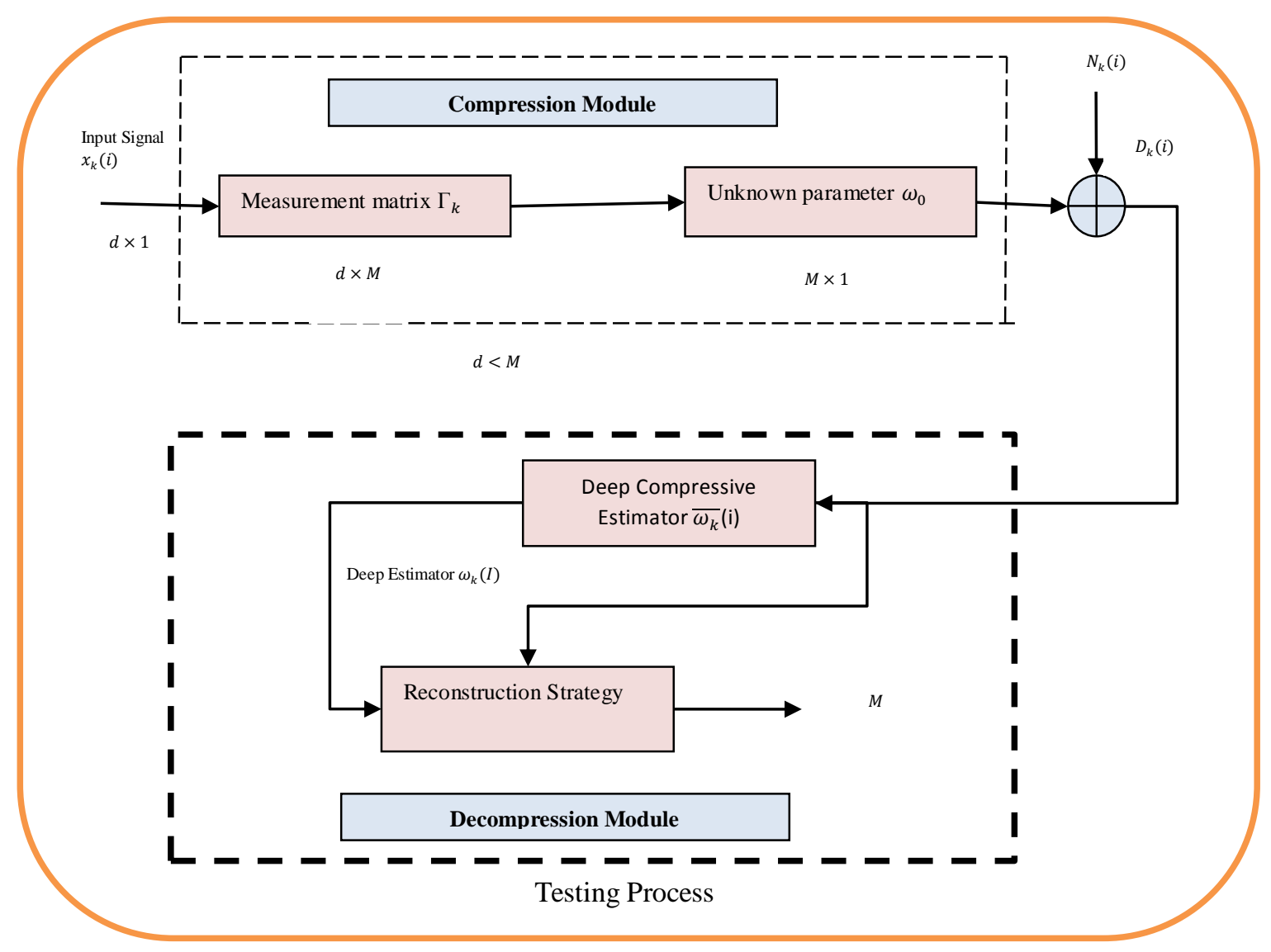

Figure 3: Proposed Compressive Sensing Modules

In the suggested technique, the sensor initially detects the $d \times$ 1 vector $\overline{x_{k}}(i)$ at every node, and then estimates $\omega_{0}$ in the compressive region with the support of the $d \times M$ measurement matrix $\Gamma$.

The scalar assessment $D_{k}(i)$ supplied by begins the explanation of the suggested deep compressive estimator technique.

$D_{k}(i)=\overline{\omega_{0}^{h}} \overline{x_{k}}(i)+n_{k}(i)$ where $\mathrm{i}=1,2$, and $\mathrm{I}$

Adaptability: At every time period $\mathrm{i}=1,2, \ldots, \mathrm{I}$, in the adaptability phase, every node $\mathrm{k}=1,2, \ldots \mathrm{N}$. Creates a locally compression estimator $\overline{\Psi_{k}}(i)$ by using :

$$
\overline{\Psi_{k}}(i)=\overline{\omega_{k}}(i)+\mu_{k}(i) e_{k}^{*}(i) \overline{x_{k}}(i)
$$

Where, $e_{k}(i)=D_{k}(i)-\overline{\omega_{0}^{h}}(i) \overline{x_{k}}(i)$

$$
\mu_{k}(i)=\frac{\mu_{0}}{\overline{x_{k}^{h}(i)} \overline{x_{k}}(i)}
$$

Data Exchange: Only the localized compression estimator $\overline{\Psi_{k}}(i)$ will be communicated among node $\mathrm{k}$ and all its neighbour nodes based on the network topology structure. Locally, the measuring matrix $\Gamma_{k}$ will be stored.

Merger: After the data sharing is completed at every time instant $\mathrm{I}=1,2, \ldots, \mathrm{I}$, the combining phase started. Through a given equation, every node will aggregate the locally compression estimation method from its adjacent nodes and itself, to calculate the compression estimator that has been modified as $\overline{\omega_{k}}(i+1)$.

$$
\overline{\omega_{k}}(i+1)=\sum_{L \in \mathbb{N}_{K}} c_{k L} \overline{\Psi_{k}}(i)
$$

This can also be utilized with other reconstructing techniques. In conclusion, only the localized compression estimator $\overline{\Psi_{k}}(i)$ will be broadcast over the network during the deep compressive estimator operation, cumulating in a reducing the number of variables to be sent from $\mathrm{M}$ to $\mathrm{d}$.

\section{Deep Learning Network Description}

The robust system is developed by application of convolution neural network $(\mathrm{CNN})$. In this work, residual learning is taken as proposed approach. For residual learning loss function is:

$$
C(\theta)=\frac{1}{2 N} \sum_{i=1}^{N}\left\|R\left(y_{i} ; \theta\right), y_{i}-x_{i}\right\|_{F}^{2}
$$

\section{Sparse and Low Rank Reconstruction}

The compressive multichannel ECG signal will be corrupted when noise penetrate the multichannel ECG signal CS system at the sender side. In this work, we examine the multichannel ECG signal CS in the existence of noise.

$$
y=\varphi x+n
$$


In terms of maximum probability, the matrix Frobenius norm is best for modeling Gaussian noise. As a result, the related optimization model is specified:

$$
\begin{gathered}
\min _{x, n, S}\|\Omega x\|_{0}+\alpha\|n\|_{f}^{2} \\
\text { S. } T y=\varphi x+n
\end{gathered}
$$

Where represents two regularization parameters $\alpha$ and $\beta$, and $\|$. $\|_{\mathrm{f}}$ signifies the matrix Frobenius norm. The optimization issue employing the $\ell_{0}$ norm, on the other hand, is strongly non-convex and generally NP-hard. We use the $\ell_{1}$ norm instead of the initial $\ell_{0}$ norm for convenience. As a result, can be rephrased as follows:

$$
\begin{gathered}
\min _{x, n, S}\|\Omega x\|_{0}+\alpha\|n\|_{f}^{2} \\
\text { S. } T y=\varphi x+n
\end{gathered}
$$

The suggested optimum framework based on sparse and low rank depiction can be written as:

$$
\begin{gathered}
\min _{x, n, S}\|\Omega x\|_{1}+\lambda * \operatorname{rank}\|x\|_{*}+\alpha * \operatorname{rank}\|n\|_{f}^{2} \\
\text { S. } T y=\varphi x+n
\end{gathered}
$$

Here $\|x\|_{*}$ denotes $\mathrm{x}$ 's nuclear norm, which is the same as the total of singular values of $\mathrm{x}$ and $\lambda$ denotes the regularization parameter.

\section{RESULT ANALYSIS}

The result analysis is performed by simulating the WBAN scenario on MATLAB platform. Three conditions are considered and discussed further: With respect to variable nodes, With respect to variable SNR, With respect to variable compression ratio. The ECG samples in our experiments are obtained from MIT-BIH Arrhythmia Database is used.

\section{Analysis with respect to variable number of Nodes}

In this section, the simulation is performed with variable number of WBAN nodes as well as with different CR. The simulation results are analyzed on three parameters such as PRD, MSE and Time. From the result it is analyzed that with increasing number of nodes the PDR decreases and time increases. The MSE was evaluated between -30 to $-40 \mathrm{db}$.

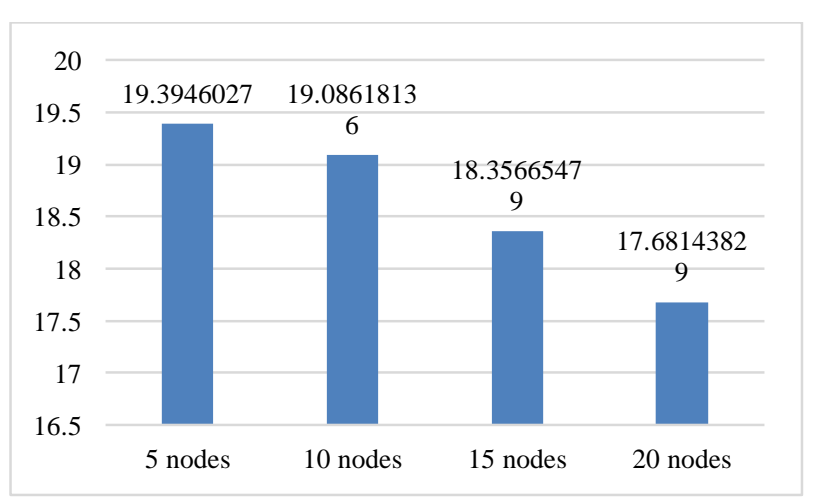

Figure 4: PRD analysis with variable nodes

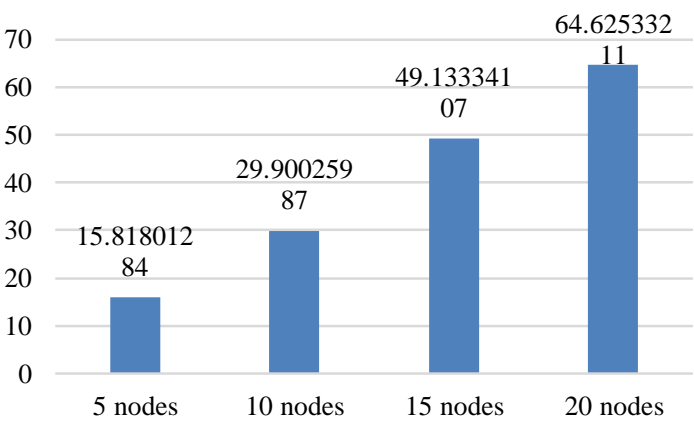

5: Time analysis with variable nodes

Figure

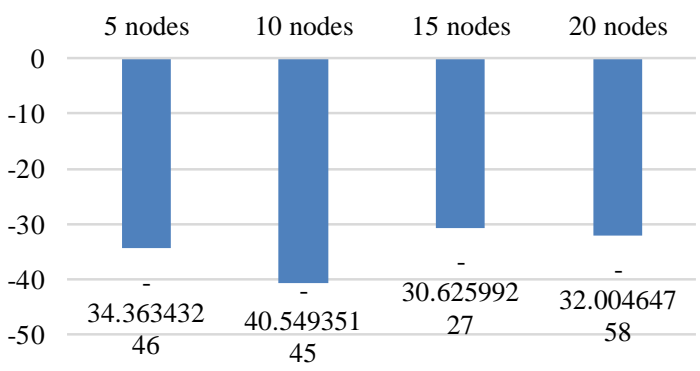

Figure 6 MSE analysis with variable nodes

\section{Analysis with respect to SNR}

In this section, the simulation is performed with variable SNR for WBAN nodes as well as with different CR. The simulation results are analyzed on three parameters such as PRD, MSE and Time. From the result it is analyzed that with increasing SNR the PDR increases. The MSE was evaluated between -30 to $-40 \mathrm{db}$ and it seems to be increasing.

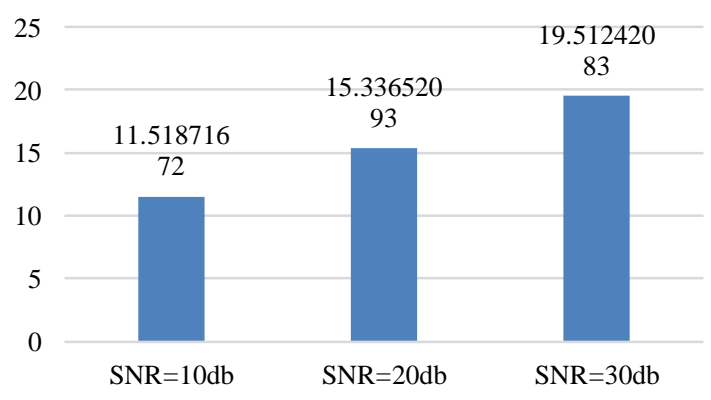

7 PRD analysis with variable $S N R$

Figure

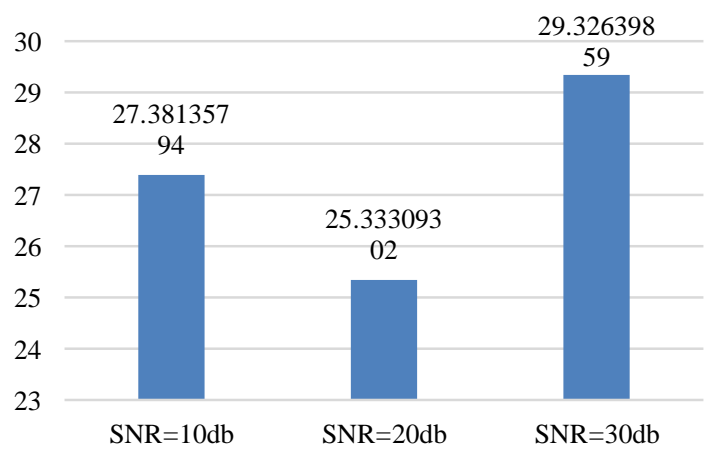

8 Time analysis with variable SNR 


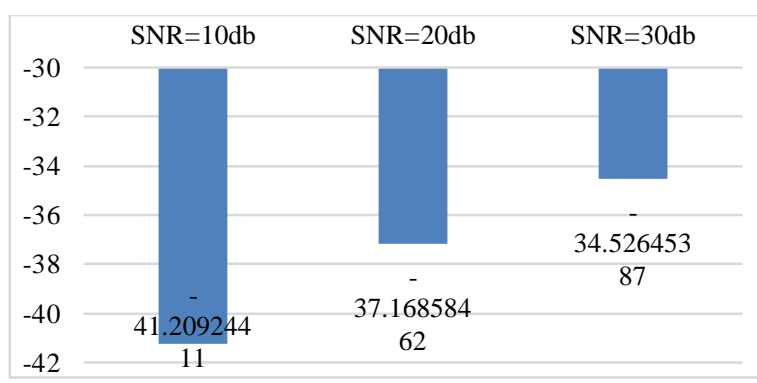

Figure 9 MSE analysis with variable SNR

Analysis with respect to compression ratio

In this section, the simulation is performed with variable $\mathrm{CR}$ for WBAN nodes as well as with different CR. The simulation results are analyzed on three parameters such as PRD, MSE and Time. The result is represented in table I.

Table I: Performance Analysis with respect to CR

\begin{tabular}{|r|r|r|r|}
\hline CR & PRD (in \%) & Time (in Sec & MSE (in db \\
\hline $\mathrm{CR}=10 \%$ & 17.8877 & 15.86288 & -35.0895 \\
\hline $\mathrm{CR}=20 \%$ & 17.76857 & 16.57276 & -34.5882 \\
\hline $\mathrm{CR}=30 \%$ & 19.57374 & 17.18017 & -33.9706 \\
\hline $\mathrm{CR}=40 \%$ & 18.79184 & 17.75342 & -35.1616 \\
\hline $\mathrm{CR}=50 \%$ & 20.45716 & 16.5836 & -33.5746 \\
\hline $\mathrm{CR}=60 \%$ & 19.67891 & 15.33226 & -33.8098 \\
\hline $\mathrm{CR}=70 \%$ & 20.79281 & 15.10771 & -34.8828 \\
\hline $\mathrm{CR}=80 \%$ & 21.38596 & 15.34359 & -34.3984 \\
\hline $\mathrm{CR}=90 \%$ & 22.11119 & 14.59247 & -34.3119 \\
\hline Average & 19.82754 & 16.03654 & -34.3882 \\
\hline
\end{tabular}

Comparative Analysis

In the research, related study aims for better signal reconstruction while reducing computation difficulty. Reconstruction algorithms, on the other hand, may have an impact on the quality of the returned signals; consequently, different reconstruction procedures are useful for comparison study. For comparison, in this work, six different methods are adopted such as OMP, basis pursuit (BP), compressive sampling matching pursuit (COSAMP), (Iteratively Reweighted Least Squares) IRLS, Least-Mean-Square Algorithm(LMS), Normalized Least-Mean-Square Algorithm (NLMS).

From result analysis, it is observed that DeepCS algorithm have lower PRD value as compared to other existing algorithms. It was observed that proposed deepCS algorithm have lower MSE (in $\mathrm{dB}$ ) as compared to other existing algorithms. For time analysis is presented that shows that deepCS algorithm requires more time for execution than other algorithms. So, it can be concluded that deepCS algorithm gives more efficient result as compared to other existing algorithms.

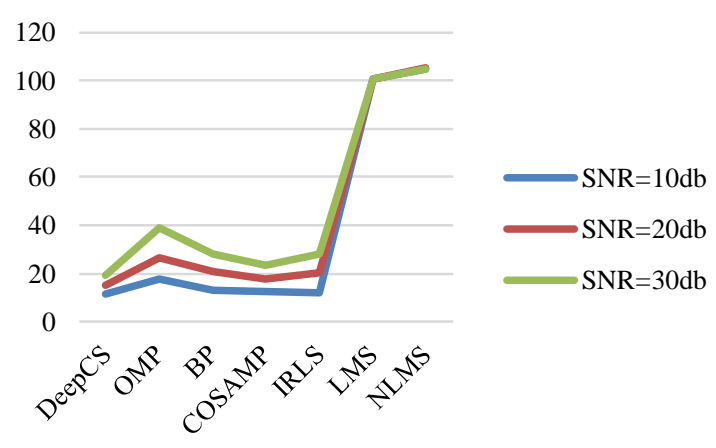

Figure 10. PRD Performance Evaluation with variation of SNR

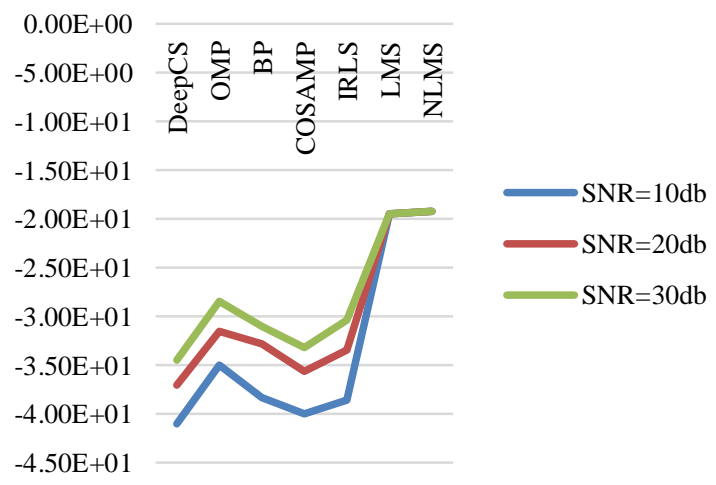

Figure 11. MSE Performance Evaluation with variation of SNR

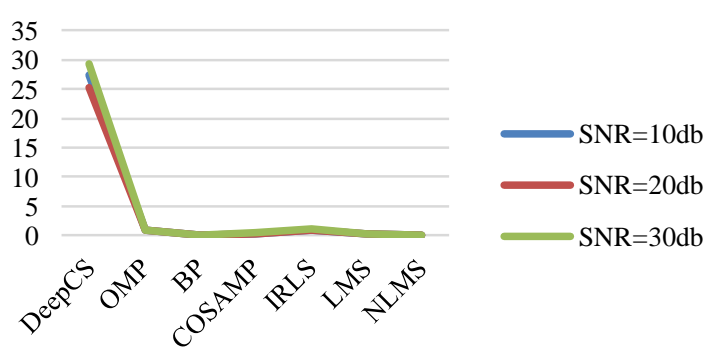

12. Time Performance Evaluation with vat

Figure

The performance of proposed deepCS is compared with existing research work presented by Zhang et al. The author presented a sparse signal recovery algorithm for recovering ECG data in the framework of CS. The model presented in this work, shows the MSE that ranges from -7 to -18 . The MSE evaluation of proposed work ranges between -30 to -40 . The comparative analysis shows and it can be concluded that proposed deepCS algorithm is more efficient as compared to existing work. 


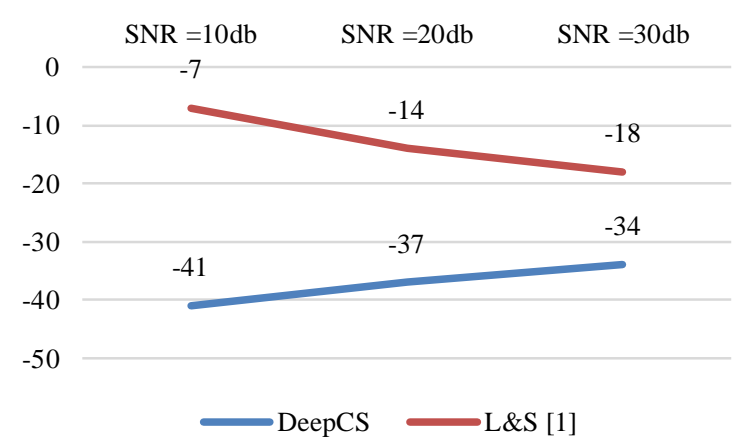

Figure 13. MSE Comparative Analysis with Existing Work

\section{CONCLUSION}

Compressive sensing (CS) has recently proven to be an excellent data compression approach for wireless body-area network tele monitoring of multichannel ECG signals. Most present EEG CS multi-channel algorithms disregard the noise. Other noise kinds, such as heavy impulsive tailed noise, are also accessible. This study proposes a deep learning-based sparse and low rank representation in the presence of noise to address the aforementioned difficulties. The simulations findings demonstrate the advantage of the deep CS offered over advanced noise strategies. The simulation is performed under different scenarios:

In first scenario, the simulation was performed with variable number of nodes and with variable compression ratios. It was observed that with increasing number of nodes the PRD decreases and the MSE was evaluated between -30 to $-40 \mathrm{db}$. In second scenario, the simulation was performed with variable SNR for WBAN nodes as well as with different CR. From the result it is analyzed that with increasing SNR the PDR increases. The MSE was evaluated between -30 to $-40 \mathrm{db}$ and it seems to be increasing. In last, the performance of proposed deepCS is compared with existing research work presented by Zhang et al. [1]. The MSE evaluation of proposed work ranges between -30 to -40 that is more efficient as compared to existing work. From result analysis of proposed work, it can be concluded that the proposed deepCS algorithm is more efficient as compared to existing work. The MSE evaluation of the proposed approach is good enough. But it is seen from the result that the proposed algorithm shows good MSE evaluation at lower SNR value and it gets increased with increased SNR. This needs to be focused for future research direction.

\section{REFERENCES}

1. Zhang, YB., Huang, LT., Li, YQ. et al. "Low-rank and joint-sparse signal recovery using sparse Bayesian learning in a WBAN", Multidim Syst Sign Process 32, 359-379 (2021). https://doi.org/10.1007/s11045020-00743-y.

2. Mamaghanian H, Khaled N, Atienza D, et al. Compressive sensing for real-time energy-efficient ECG compression on wireless body sensor nodes. IEEE T Bio-Med Eng 2011; 58(9): 2456-2466.

3. Brunelli D and Caione C. Sparse recovery optimization in wireless sensor networks with a sub-Nyquist sampling rate. Sensors $2015 ; 15(7)$ : $16654-16673$.

4. Majumdar A and Ward RK. Energy efficient EEG sensing and transmission for wireless body area networks: a blind Compressive sensing approach. Biomed Signal Proces 2015; 20: 1-9.
5. Mangia M, Bortolotti D, Pareschi F, et al. Zeroing for HW-efficient compressed sensing architectures targeting data compression in wireless sensor networks. Microprocess Microsy 2017; 48: 69-79.

6. Peng H, Tian Y, Kurths J, et al. Secure and energyefficient data transmission system based on chaotic compressive sensing in body-tobody networks. IEEE T Biomed Circ S 2017; 11(3): 558-573.

7. S. Sawant, S. Banerjee and S. Tallur, "Compressive sensing based dataloss recovery enables robust estimation of damage index in ultrasonic structural health monitoring," 2020 IEEE SENSORS, 2020, pp. 1-4, doi: 10.1109/SENSORS47125.2020.9278599.

8. G. J. A and T. A. C., "Signal recovery from random measurements via orthogonal matching pursuit," IEEE Trans. Info. Theory, vol. 53(12), pp. 4655-4666, 2007

9. Tirthankar Sengupta, Shivi Jain, Mani Bhushan, "A Compressive sensing Based Basis-pursuit Formulation of the Room Algorithm, IFAC Proceedings, Volume 46, Issue 31, 2013, Pages 238-243.

10. D. Needell and J. Tropp, "CoSaMP: Iterative signal recovery from incomplete and inaccurate samples," Applied and Computational Harmonic Analysis, pp. 301-321, 2009.

11. R. Chartrand and Wotao Yin, "Iteratively reweighted algorithms for compressive sensing," 2008 IEEE International Conference on Acoustics, Speech and Signal Processing, 2008, pp. 3869-3872, doi: 10.1109/ICASSP.2008.4518498.

12. Li, Yingsong and M. Hamamura. "An Improved Proportionate Normalized Least-Mean-Square Algorithm for Broadband Multipath Channel Estimation." The Scientific World Journal 2014 (2014). 